\title{
THE MINIMUM NUMBER OF GENERATORS FOR INSEPARABLE ALGEBRAIC EXTENSIONS ${ }^{1}$
}

\author{
M. F. BECKER AND S. MACLANE
}

1. Finite algebraic extensions of imperfect fields. A finite separable algebraic extension $L$ of a given field $K$ can always be generated by a single primitive element $x$, in the form $L=K(x)$. If $K$ has characteristic $p$, while $L / K$ is inseparable, there may be no such primitive element. The necessary and sufficient condition for the existence of such an element is to be found in Steinitz. ${ }^{2}$ When there is no such primitive element, there is the question $:^{3}$ given $K$, what is the minimum integer $m$ such that every finite extension $L / K$ has a generation $L=K\left(x_{1}, x_{2}, \cdots, x_{m}\right)$ by not more than $m$ elements?

The question can be answered by employing Teichmüller's ${ }^{4}$ notion of the "degree of imperfection" of $K$. In invariant fashion, a field $K$ of characteristic $p$ determines a subfield $K^{p}$ consisting of all $p$ th powers of elements of $K$. If the extension $K / K^{p}$ is finite, its degree $\left[K: K^{p}\right]$ is a power $p^{m}$ of the characteristic, and the exponent $m$ is called the degree of imperfection of $K$. For instance, let $P$ be a perfect field of characteristic $p$ and let $x, y$ be elements algebraically independent with respect to $P$. Form the fields

$$
S=P(x), \quad T=P(x, y) .
$$

Then $S=S^{p}(x),\left[S: S^{p}\right]=p$, while $\left[T: T^{p}\right]=p^{2}$, so that $T$ is "more imperfect" than $S$.

THEOREM 1. If the field $K$ of characteristic $p$ has a finite degree of imperfection $m$, then every finite algebraic extension $L \supset K$ can be obtained by adjoining not more than $m$ elements to $K$. Furthermore, there exist finite extensions $L \supset K$ which cannot be obtained by adjoining fewer than $m$ elements to $K$.

Proof. First consider the particular extension $K^{1 / p}$ consisting of all $p$ th roots of elements in $K$. Because of the isomorphism $a \leftrightarrow a^{1 / p}$,

$$
\left[K^{1 / p}: K\right]=\left[K: K^{p}\right]=p^{m} .
$$

Each element $y$ in $K^{1 / p}$ satisfies over $K$ an equation $y^{p}=a$ of degree $p$.

\footnotetext{
1 Presented to the Society, October 28, 1939.

2 E. Steinitz, Algebraische Theorie der Körper, Berlin, de Gruyter, 1930, p. 72.

${ }^{3}$ This problem was suggested to one of us by $O$. Ore.

${ }^{4}$ O. Teichmüller, p-Algebren, Deutsche Mathematik, vol. 1 (1936), pp. 362-388.
} 
If $K^{1 / p}$ had generators $y_{1}, \cdots, y_{n}$ in number less than $m$, the degree $\left[K^{1 / p}: K\right]$ could not exceed $p^{n}$, a contradiction to (2).

An explicit generation for $K / K^{p}$ can be found by successively choosing elements $x_{i}$ of $K$ such that each $x_{i}$ is not in $K^{p}\left(x_{1}, \cdots, x_{i-1}\right)$. Then each $x_{i}$ satisfies an irreducible equation ${ }^{5} \quad x_{i}{ }^{p}=a_{i}$ over $K^{p}\left(x_{1}, \cdots, x_{i-1}\right)$. The adjunction of $x_{i}$ is an extension of degree $p$; so

$$
K=K^{p}\left(x_{1}, x_{2}, \cdots, x_{m}\right), \quad\left[K: K^{p}\right]=p^{m},
$$

where $m$ is the degree of imperfection ${ }^{6}$ of $K$.

Now let $L$ be any finite extension of $K$. Because of the isomorphism $a \leftrightarrow a^{p}$, one has $[L: K]=\left[L^{p}: K^{p}\right]$. Hence

$$
\left[L: L^{p}\right]=[L: K] \cdot\left[K: K^{p}\right] /\left[L^{p}: K^{p}\right]=\left[K: K^{p}\right]=p^{m} .
$$

Therefore $K$ and $L$ have the same degree of imperfection. But $L$ has an explicit generation $L=L^{p}\left(y_{1}, \cdots, y_{m}\right)$ like that of (3). If $L^{p^{n}}$ denotes the field of all $p^{n}$ th powers of elements of $L$, the isomorphism $a \leftrightarrow a^{p^{n}}$ yields $L^{p^{n}}=L^{p^{n+1}}\left(y_{1}^{p^{n}}, \cdots, y_{m^{p^{n}}}\right)$. By an induction on $n$,

$$
L=L^{p^{n}}\left(y_{1}, \cdots, y_{m}\right) \text {. }
$$

Since $L / K$ is finite, there is an integer $n$ so large that for each $y$ in $L$ the power $y^{p^{n}}$ is separable over $K$. The separable extension ${ }^{7} K\left(L^{p^{n}}\right) / K$ has a single generator $K\left(L^{p^{n}}\right)=K\left(y_{0}\right)$. Since $y_{0}$ is separable, the usual theorem ${ }^{8}$ of the primitive element yields a single element $y^{\prime}$ such that $K\left(y_{0}, y_{1}\right)=K\left(y^{\prime}\right)$. Thus, by (5),

$$
L=K\left(y_{0}, y_{1}, y_{2}, \cdots, y_{m}\right)=K\left(y^{\prime}, y_{2}, \cdots, y_{m}\right) .
$$

This is a generation by $m$ elements, as required.

The degree of imperfection of a field $K$ may be infinite, in the sense that the extension $K / K^{p}$ used in the definition is infinite. Our arguments in this case give the following result.

THEOREM 2. If the degree of imperfection of a field $K$ is infinite, then for each integer $n>0$ there exists a finite algebraic extension $L \supset K$ which cannot be obtained by adjoining fewer than $n$ elements to $K$.

\footnotetext{
${ }^{5}$ For the usual properties of such equations, cf. A. A. Albert, Modern Higher Algebra, chap. 7.

${ }^{6}$ The set $\left\{x_{1}, \cdots, x_{m}\right\}$ of independent generators is called a $p$-basis for $K$. See O. Teichmüller, loc. cit., §3, or S. MacLane, Modular fields, I. Separating transcedence bases, Duke Mathematical Journal, vol. 5 (1939), pp. 372-393.

${ }^{7}$ Here $K\left(L^{p^{n}}\right)$ denotes the field obtained from $K$ by adjoining all elements of the field $L^{p^{n}}$.

${ }^{8}$ B. L. van der Waerden, Moderne Algebra, vol. 1, 1st edition, §34. Cf. also Steinitz, loc. cit., p. 72.
} 
It might be thought that the minimum number of generators for an extension $L / K$ is related to $t$, the transcendence degree of $K$ over its maximum perfect subfield. However, this degree $t$ may be larger than the degree of imperfection $m$. For a power series field $K$, Teichmüller observed that $m=1$, while $t$ is infinite. Even when $t$ and $m$ are both finite, they can differ, as one of us showed by a more involved example ${ }^{9}$ with $t=2, m=1$.

2. Infinite algebraic extensions of imperfect fields. In applying our criterion for the minimum number of generators one needs to compute the degree of imperfection of a given field. A perfect field contains $p$ th roots of all of its elements, hence has degree of imperfection zero. A simple transcendental extension $K(t)$ has a degree of imperfection one greater than the degree of imperfection of $K$, as Teichmüller has proved (cf. also the examples (1)). On the other hand, the computation (4) proves the following theorem.

THEOREM 3. The degree of imperfection of a field is not changed by a finite algebraic extension.

There remains the case of an infinite algebraic extension $L / K$. Such an extension is purely inseparable (or, a "radical" extension) if for each element $a$ of $L$ some power $a^{p^{c}}$ lies in $K$. In this case we have the following result.

THEOREM 4. If $K$ has a finite degree of imperfection $m$, then the degree of imperfection of a purely inseparable infinite extension of $K$ is less than $m$, the degree of imperfection of $K$.

Let $L$ be a purely inseparable, infinite extension of $K$. We use a chain of intermediate fields

$$
K \subset L_{1} \subset L_{2} \subset L_{3} \subset \cdots \subset L_{n} \subset \cdots \subset L,
$$

where $L_{n}$ consists of all elements of $L$ with $p^{n}$ th power in $K$. The field $L_{n+1}$ is obtained from $L_{n}$ by adjoining $p$ th roots of a sufficient number of elements of $L_{n}$. By (4), the degree of imperfection of each $L_{n}$ is $m$. Hence, $L_{n+1}$ is a field of degree at most $p^{m}$ over $L_{n}$. Since $\left[L_{n}: K\right]$ is then finite, each $L_{n+1}$ is larger than the preceding $L_{n}$.

By the definition of the tower (6), each $L_{n} \supset L_{n+1}^{p}$. Since $L^{p} \supset L_{n+1}^{p}$, any element $\alpha$ of $L_{n}$ has over $L_{n+1}^{p}$ a degree ${ }^{10}\left[\alpha: L_{n+1}^{p}\right] \geqq\left[\alpha: L^{p}\right]$. In other words,

${ }^{9}$ S. MacLane, loc. cit., $\$ 10$.

${ }^{10}$ In fact, $\left[\alpha: L_{n+1}^{p}\right]=\left[\alpha: L^{p}\right]$. Since $\alpha$ is an element of $L_{n},\left[\alpha: L_{n+1}^{p}\right]=p$ or 1 , hence $\left[\alpha: L^{p}\right]=p$ or 1 . If $\left[\alpha: L^{p}\right]=1, \alpha^{p^{-1}}$ is in $L$ and $\left(\alpha^{p^{-1}}\right)^{p^{n+1}}=\alpha^{p^{n}}$ is in $K$. Thus, by definition of $L_{n+1}, \alpha^{p^{-1}}$ is in $L_{n+1}$, so $\left[\alpha: L_{n+1}^{p}\right]=1$. 


$$
\left[L^{p}\left(L_{n}\right): L^{p}\right] \leqq\left[L_{n+1}^{p}\left(L_{n}\right): L_{n+1}^{p}\right] .
$$

But $L_{n+1}^{p}\left(L_{n}\right)=L_{n}$, while $L_{n+1}>L_{n}$ and $\left[L_{n+1}^{p}: L_{n}^{p}\right]>1$. Thus

$$
\left[L^{p}\left(L_{n}\right): L^{p}\right] \leqq\left[L_{n}: L_{n+1}^{p}\right]<\left[L_{n}: L_{n+1}^{p}\right] \cdot\left[L_{n+1}^{p}: L_{n}^{p}\right]=\left[L_{n}: L_{n}^{p}\right] .
$$

This degree $\left[L_{n}: L_{n}^{p}\right]$ is simply $p^{m}$, with $m$ the degree of imperfection of $L_{n} ;$ so

$$
\left.{ }_{[} L^{p}\left(L_{n}\right): L^{p}\right] \leqq p^{m-1} .
$$

The maximum value of these degrees in (7) thus determines an integer $\delta \leqq m-1$ with

$$
p^{\delta}=\max \left[L^{p}\left(L_{n}\right): L^{p}\right], \quad \delta<m .
$$

We assert that $\delta$ is the degree of imperfection of $L$. In the first place, $L \supset L^{p}\left(L_{n}\right)$; so $\left[L: L^{p}\right] \geqq\left[L^{p}\left(L_{n}\right): L^{p}\right]=p^{\delta}$, where we have so chosen $n$ as to give the maximum in (8). If, however, $\left[L: L^{p}\right]$ exceeds $p^{\delta}$, there must be $\delta+1$ elements $a_{0}, a_{1}, \cdots, a_{\delta}$ in $L$ such that

$$
\left[L^{p}\left(a_{0}, a_{1}, \cdots, a_{\delta}\right): L^{p}\right]=p^{\delta+1},
$$

contrary to the definition (8) of $\delta$. Thus $\delta$, the degree of imperfection of $L$, is less than the corresponding degree of imperfection for $K$.

The degrees used in the computation (8) of $\delta$ can be expressed explicitly by choosing a $p$-basis $x_{1}, \cdots, x_{m}$ for each $L_{n}$, for then

$$
\left[L^{p}\left(L_{n}\right): L^{p}\right]=\left[L^{p}\left(L_{n}^{p}\left(x_{1}, \cdots, x_{m}\right)\right): L^{p}\right]=\left[L^{p}\left(x_{1}, \cdots, x_{m}\right): L^{p}\right] .
$$

Consider now an infinite extension $L / K$ which is not purely inseparable, and let $M$ denote the field of all elements of $L$ separable over $K$. Even if $M / K$ is infinite, $M$ and $K$ still have the same degree of imperfection, according to a result of Teichmüller. ${ }^{11}$ If the exponent of $L$ is taken to be the least integer $e$ such that all powers $a^{p^{e}}$ of elements $a$ in $L$ are separable over $K$, we then have the following theorem.

THEOREM 5. If $K$ has a finite degree of imperfection, then an algebraic extension $L$ of $K$ has the same or a smaller degree of imperfection according as $L$ has a finite or an infinite exponent over $K$.

3. Generators for given extensions. In $\$ 1$ we determined the minimum number of generators for all algebraic extensions of a fixed base field. Suppose, however, $L$ is a specific extension of $K$. We wish to

${ }^{11}$ Any $p$-basis for $K$ is also a $p$-basis for an arbitrary separable algebraic extension $M$ of $K$; cf. Teichmüller, loc. cit., p. 170. 
get the minimum number of generators for this particular extension. It clearly suffices to consider $L$ a purely inseparable finite extension of $K$.

Whether the degree of imperfection of $L$ is finite or infinite there is a subset $U$ in $L$ such that $L^{p}\left(U^{\prime}\right)=L$. By the same argument as in $\$ 1$

$$
L=L^{p^{n}}(U)
$$

for integral $n$.

Consider, now, the field $L^{p}(K)$ between $L$ and $K$. For $L / K$ finite, $L / L^{p}(K)$ is finite and $\left[L: L^{p}(K)\right]=p^{r}$. Since the $p$ th power of every element in $L$ is contained in $L^{p}(K), r$ elements $X_{1}, X_{2}, \cdots, X_{r}$ in $L$ can be chosen such that

$$
L=L^{p}(K)\left(X_{1}, \cdots, X_{r}\right)=L^{p}\left(K, X_{1}, X_{2}, \cdots, X_{r}\right) .
$$

If $e$ is the exponent of $L / K$, using (9) we obtain

$$
L=L^{p t}\left(K, X_{1}, \cdots, X_{r}\right)=K\left(X_{1}, X_{2}, \cdots, X_{r}\right),
$$

Hence $L / K$ can be generated by $r$ elements.

Moreover, $r$ is the minimum number of generators. For if $L=K\left(Y_{1}, \cdots, Y_{s}\right)$ where $s<r$,

$$
\begin{aligned}
L^{p} & =K^{p}\left(Y_{1}^{p}, \cdots, Y_{s}^{p}\right), \quad L^{p}(K)=K\left(Y_{1}^{p}, \cdots, Y_{s}^{p}\right), \\
p^{r} & =\left[L: L^{p}(K)\right]=\left[K\left(Y_{1}, \cdots, Y_{s}\right): K\left(Y_{1^{p}}, \cdots, Y_{s}^{p}\right)\right] \leqq p^{s},
\end{aligned}
$$

and $r \leqq s$, against assumption.

TheOREm 6. If $L$ is a purely inseparable finite extension of $K$, the minimum number of generators of $L / K$ is $r$, the exponent determined by the degree $\left[L: L^{p}(K)\right]=p^{r}$.

NeW York, N.Y., AND HARVARD UNIVERSITY 